\title{
An Expert System Approach for Analyzing and Evaluating the Testing Results of CTCS-3 On-board Equipment
}

\author{
Yong Zhang ${ }^{1, a}$, Yuxi Yang ${ }^{2, b}$ \\ ${ }^{1}$ School of Electronic and Information Engineering, \\ Beijing Jiaotong University, Beijing 100044, China; \\ ${ }^{2}$ School of Electronic and Information Engineering, \\ Beijing Jiaotong University, Beijing 100044, China. \\ azhangy@bjtu.edu.cn, b13120291@bjtu.edu.cn
}

Keywords: on-board equipment, CTCS-3, train control system, testing results, analysis and evaluation, expert system, rule representation

\begin{abstract}
The on-board equipment is an important part of CTCS-3 train control system. In order to verify whether the on-board equipment complies with CTCS-3 system requirements specification, it is necessary to carry out functional testing in laboratory, in which a lot of professional expertise is needed, especially in the analysis and evaluation of the testing results. Therefore, it makes sense to carry out the research on the method for analyzing and evaluating of testing results based on expert system. By establishing a rule base based on production rule representation for rule inference and comparing the rule data with the testing data gathered from various source such as DMI and data recorder, the analysis and evaluation method can be realized. Several key issues such as rule representation, testing data extraction and comparison strategy are addressed in this paper.
\end{abstract}

\section{Introduction}

The CTCS-3 train control system which utilizes the GSM-R radio communication to realize train-track data transmission is the key technical equipment to ensure the safety, reliability and high efficiency of train operation at speed above $300 \mathrm{~km} / \mathrm{h}$. A CTCS-3 system consists of the ground equipment and the on-board equipment. The on-board equipment is responsible for receiving commands from the ground equipment and generating the speed control curve to supervise train operation. Therefore, it is necessary to carry out a series of testing to verify whether the on-board equipment complies with related technical specifications [1-3] before putting it into service.

For the laboratory testing of the onboard equipment, the black-box method based on data-driven mechanism is usually adopted [4]. Before the testing, a set of test data shall be prepared. During the testing, the testers shall observe and record the testing results at the visible interfaces such as DMI (Driver Machine Interface). Finally manual analysis is required for analyzing and evaluating the testing results, by comparing the expected results of the test data with the testing results gathered from different sources such as DMI display and data recorder.

At present, the analysis and evaluation of testing results are done manually, which is time-consuming, low efficiency and with high demand for expertise. Therefore, it is necessary to do some search to automate the analysis and evaluation work.

Some research has been carried out at home and abroad. MULTITEL has provided complete solutions for automated testing of ERTMS on-board equipment, to realize a semi-automatic analysis and validation of the testing results [5]. Some preliminary work has been done at home, concerning the automatic recognition of DMI [6], automatic analyzing of testing results [7].

This paper focuses on the analysis and evaluation of the testing results based on an expert system approach. It is organized as follows: firstly the functional testing of CTCS-3 on-board equipment is briefly explained; secondly the rule source and rule representation method are introduced; thirdly the process of analyzing and evaluating of the testing results, involving data extraction, comparison 
between rules and testing results as well as results evaluation are elaborated; finally a conclusion is drawn and the future works are envisaged.

\section{CTCS-3 On-board Equipment Testing in Brief}

Structure of On-board Equipment. The main function of the CTCS-3 on-board equipment is to supervise train operation based on train positioning, and train control related information sent from the trackside equipment.

The on-board equipment consists of: Vital Computer(VC), Track Circuit Receiving module (TCR), Radio Transmission Module (RTM), Balise Transmission Module (BTM), Driver Machine Interface (DMI), Train Interface Unit (TIU), Juridical Recording Unit (JRU) and etc. The VC processes the data from other modules and calculates speed control profile for supervising train operation; the DMI display various kinds of train control information to the driver; BTM, RTM and TCR receive and process trackside data and send relevant information to VC; JRU records all information related with train operation and equipment status[1].

Operating Mode of On-board Equipment. The working process of CTCS-3 on-board equipment is achieved through the transition of its operating modes which include Full Supervision (FS), On Sight (OS), Calling On (CO), Shunting (SH), Isolation (IS), Stand By (SB), Sleeping (SL), Trip (TR), and Post Trip (PT) [1].

Functional Testing of On-board Equipment. The functional testing of CTCS-3 on-board equipment is kind of a black-box testing. The testing platform of on-board equipment is shown as in Fig.1 [8].

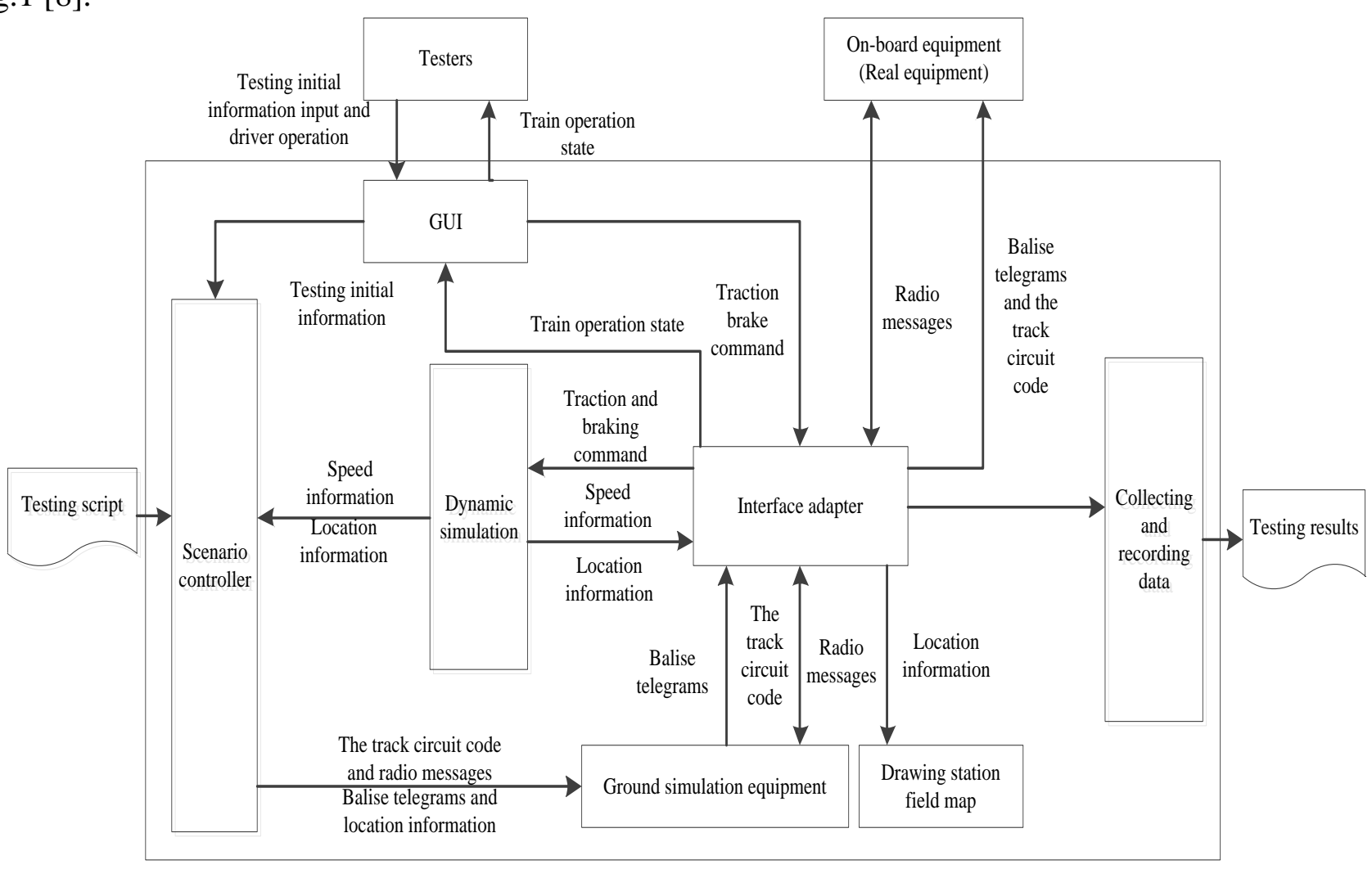

Fig.1 Architecture of On-board Equipment Testing Platform

The testing platform consists of five functional modules: Scenario Controller, Trackside Simulator, Train Dynamics Simulator, GUI, Interface Adapter, and Data Collector. The scenario controller is in charge of controlling the testing process; Train Dynamics Simulator is responsible for simulating train motion dynamics; the Trackside Simulator sends the necessary trackside information to the on-board simulator; the Data Collector gathers all the data produced during the testing process, the GUI provides a graphic interface for the testers to monitor and control the test process. 


\section{Rule Source and Rule Representation}

Rule Source. For CTCS-3 on-board equipment, all rules are extracted from the "System Requirements Specification for CTCS-3 Train Control System (v1.0)"[1], "General Technical Scheme of CTCS-3 Train Control System"[2], as well as the summary of testing experiences and expert knowledge.

Rule Representation. The production rule representation is a commonly used knowledge representation method, which is suitable for representing structural knowledge. A production rule generally has an antecedent representing the conditions that must be satisfied; it also has a consequence that represents the conclusion or operation after applying the rule. The structure of a production rule is as follows.

IF < condition > THEN < conclusion> or IF < condition > THEN < operation>

Two examples are given as follows.

1) In the RBC/RBC handover scenario, the on-board equipment shall send train data to the governing RBC after it has established a safe radio link with the RBC and it is not in Sleeping Mode.

The rule of this requirement can be expressed as follows.

IF (Link_Status==1\&\&Mode! $=$ SL)

THEN (_ToRBC.NID_MESSAGE==129\&\&_NID_PACKET==11)

2) In the operating mode transition scenario, the on-board equipment shall change into Standby mode when it is currently in Full supervision mode and the driver desk is closed.

The production rule for this requirement is as follows.

IF (Mode $==$ FS\&\&DriverDesk_Status $==0 \times 0$ )

THEN (Mode==SB)

Rule Storage. Due to the complexity of the test specification, the number of rules is too large to be stored in one single table. Therefore, the rules are classified into different rule tables, according to different test contents, and stored in a rule base. The structure of the rule base is shown as in Fig. 2.

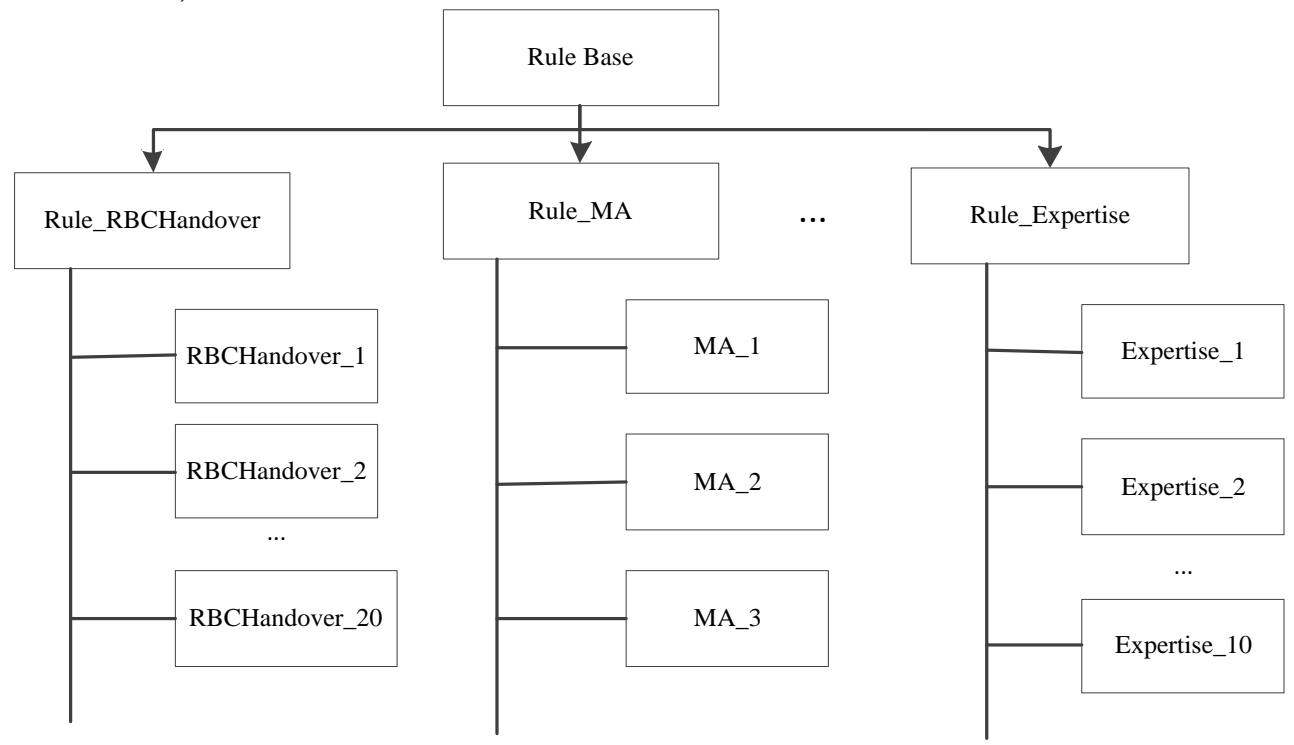

Fig. 2 Structure of the Rule Base

\section{Analysis and Evaluation of testing results}

Whole Process. The key issues in the analysis and evaluation of testing results include data extraction, comparison strategy and testing results evaluation. The whole process is shown as in Fig. 3. 


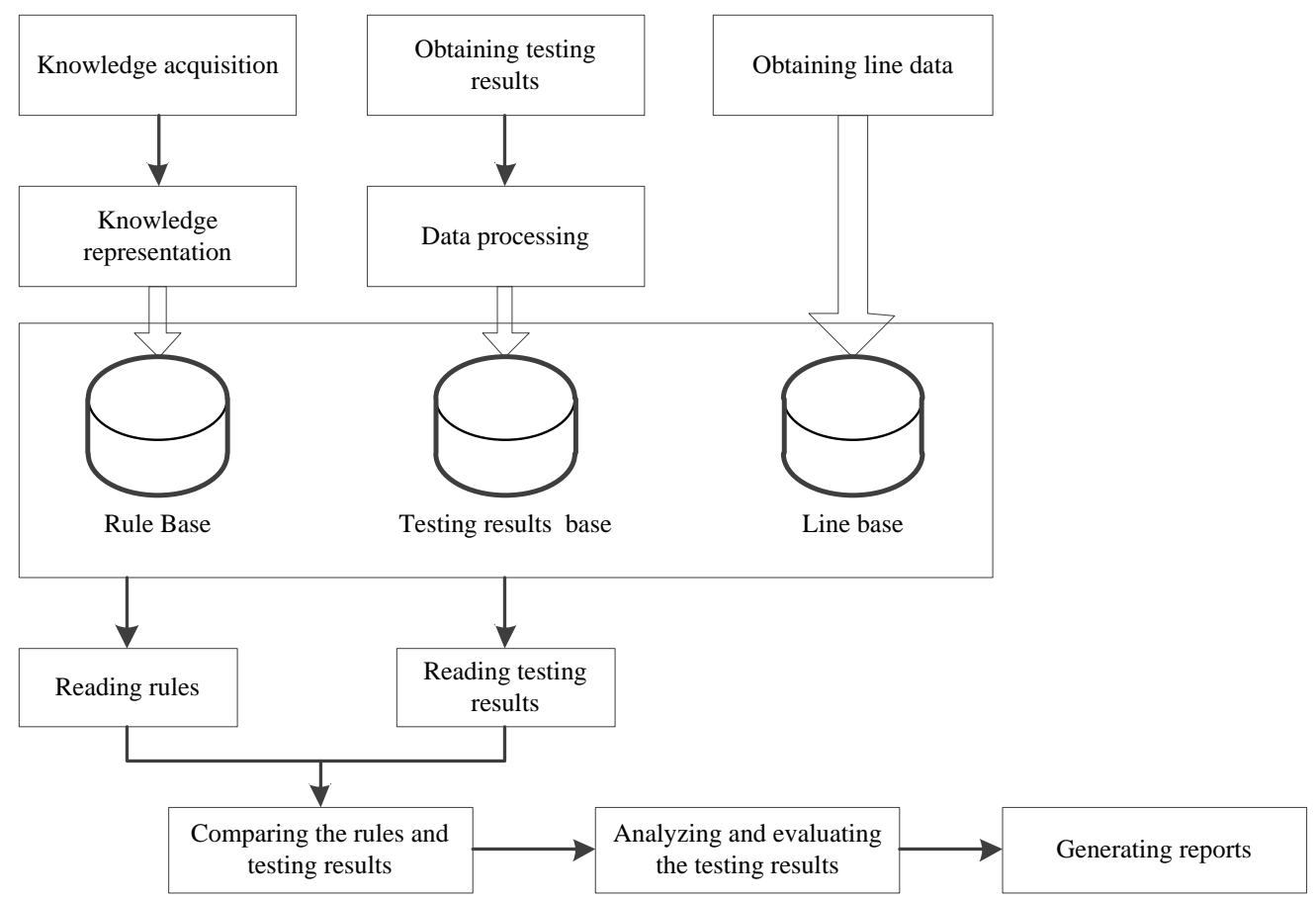

Fig. 3 The whole process of testing results analyzing and evaluating

Data Extraction. Analysis and evaluation of testing results based on expert system platform needs data supporting, which consists of testing results, line data and data of test specifications according to different sources, with relationships shown as in Fig. 4.

Influence: when preparing the test specification data, the line data will be modified in some test scenarios to meet the laboratory test conditions, for example, rewriting the Balise message of corresponding line or track circuit length.

Integration: line data is embedded in test specifications data after rewriting line data in the actual test.

Decision: testing data from on-board equipment testing platform is outputted based on line data and test specification data.

Reflection: the recorded test data is the reaction of line data and test specification data.

Testing results refer to the data obtained from an actual operation and they are mainly acquired from DMI and JRU module. Line data is a set of fixed line data according to different lines and different scenarios. Data of test specifications is data-oriented requirements specification and it has been introduced above.

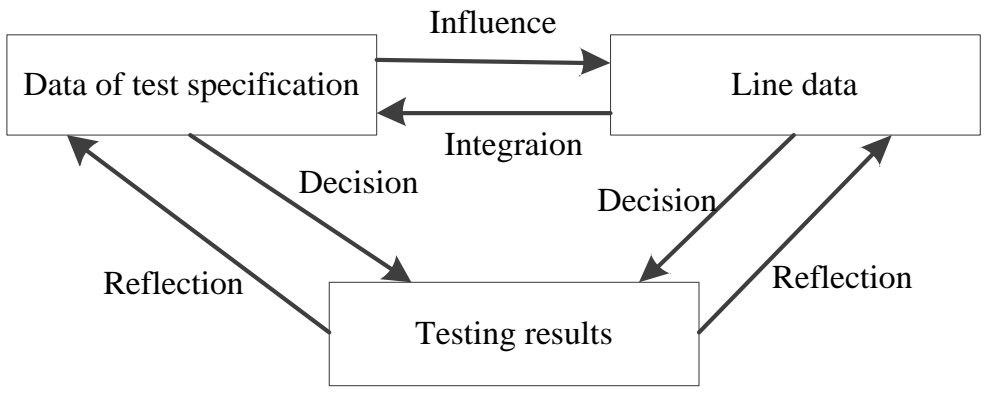

Fig. 4 Relationship of testing data

The interface associated with the on-board equipment include DMI (Driver-Machine Interface), JRU (Judicial Recorder Unit), BTM (Balise Transmission Module), RTM (Radio Transmission Module), TCR (Track Circuit Reader) [1].

Testing results are acquired largely from DMI by image recognition, and supplemented by data from JRU, RTM and BTM. The data acquisition path is shown as in Fig. 5. 


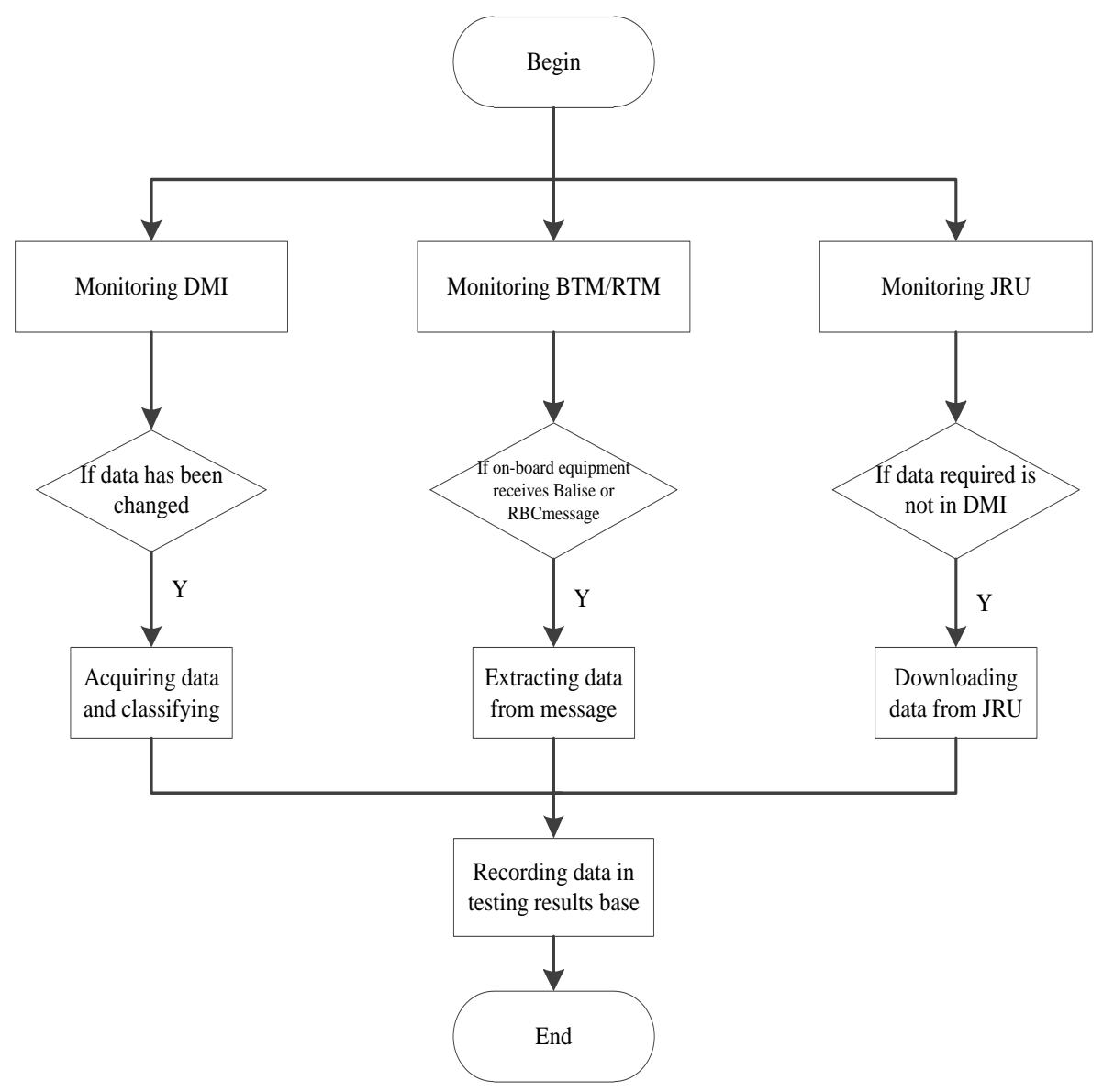

Fig. 5 Data acquisition path

In order to understand the state of the on-board equipment during the test, we need to focus on the data below:

State information: describing operating status of the on-board equipment, including application level and operating mode, brake status, emergency message, etc.

Train status information: including current speed, permitted speed, train position, etc.

Messages from Balise and RBC: including Movement Authority (MA), line speed, gradient, Temporary Speed Restriction (TSR), automatic passing phase-separation, RBC/RBC handover, etc.

Line data usually contain the information about station, line speed, gradient, track section, etc. Comparison strategy. Comparison strategy refers to the method used to compare the testing results with the rule. Firstly, selecting the rules from the rule base form a rule set. Secondly, separating the conditions from the conclusions of the rules in the rule set. Thirdly, comparing the condition with the initial condition of the testing results and the conclusions with the terminal state of the testing results. To realize comparison process, a stack is used for storing the conditions and conclusions of the rules. If all the conditions and conclusions of a rule have been pushed into the stack, it means that the rule is excuted successfully.The process of the comparison is shown as in Fig. 6. 


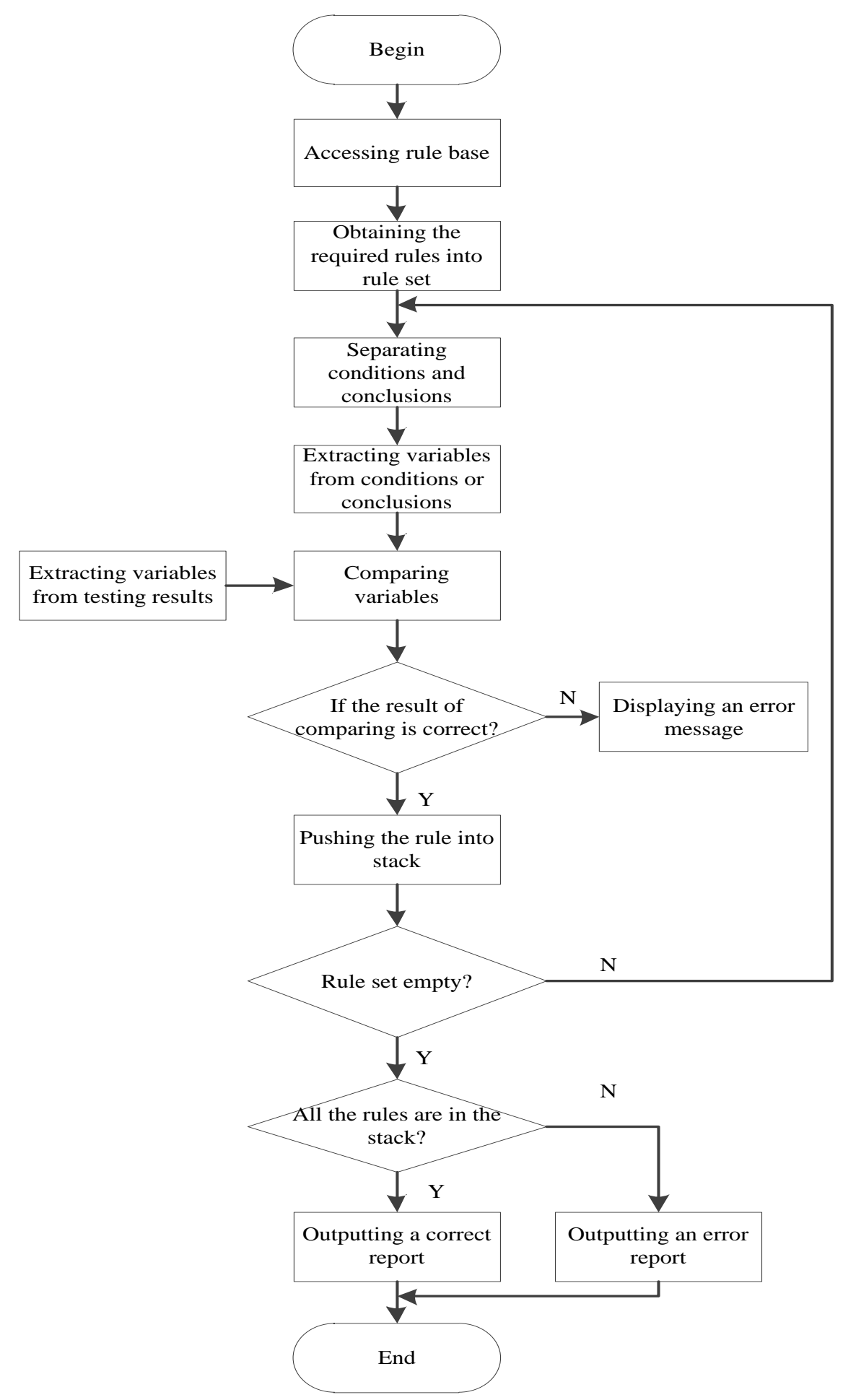

Fig. 6 Process of the comparison strategy

Testing Results Evaluation. In one test, it is successful only if all the steps and states meet the system requirement specification. We can design evaluation function to validate the correctness of the actual testing results. The expert system will put the results of comparing into the evaluation function automatically after analyzing. If the result of evaluating is incorrect, a prompt message will be generated. After all the evaluation steps have been completed, the expert system will generate an analysis and evaluation report which includes the name of test script, the name of test line and the analysis and evaluation results of each step. To improve the efficiency, we parallelize the evaluation in one step with analyzing in next step by multi-threading. The process of evaluating testing results is shown as in Fig. 7. 


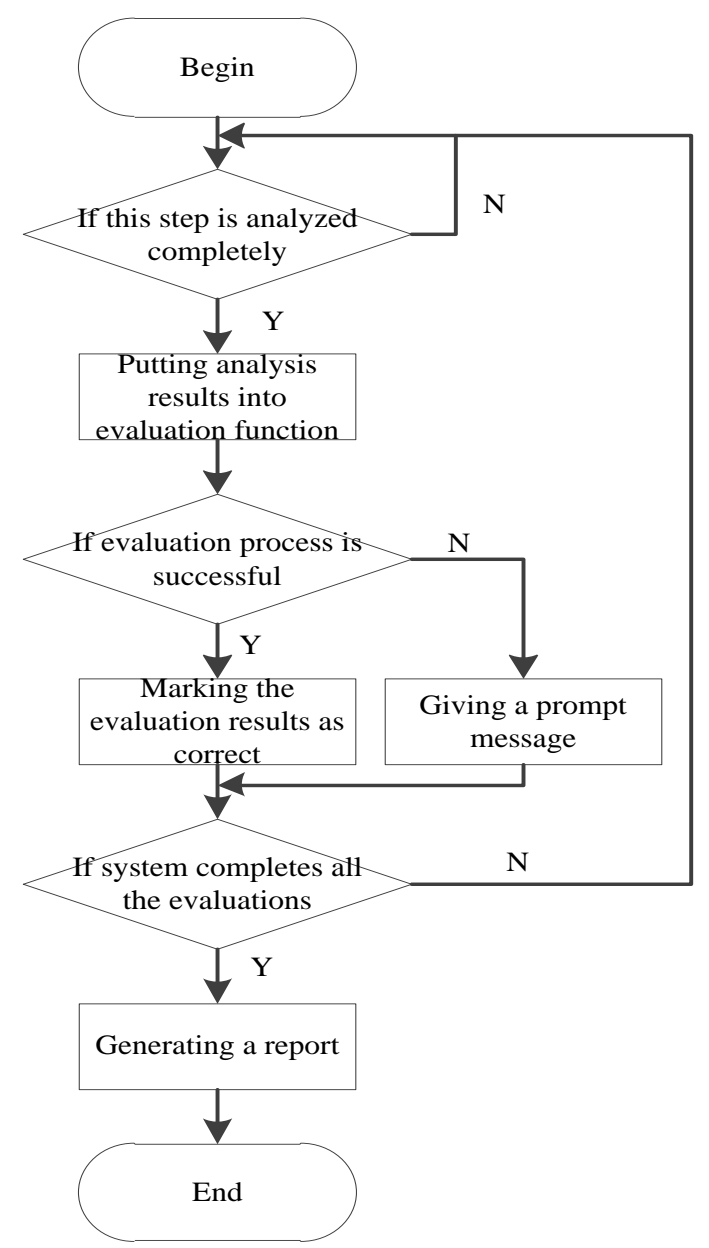

Fig. 7 Process of evaluating testing results

\section{Conclusion and Future Works}

This paper proposes a method for analyzing and evaluating the testing results of CTCS-3 on-board equipment based on expert system. A rule base based on production rule representation is established. Solutions for data extraction, comparison strategy and evaluating testing results are proposed. Future works include the automatic extraction of the rule from the rule base and the study on optimization of analysis process.

\section{Acknowledgement}

This work was supported in part by the Nature Science Foundation under Grant 61490705.

\section{References}

[1]. Transportation Bureau of MOR, "System Requirements Specification for CTCS-3 Train Control System (v1.0),” Beijing: China Railway Press, Beijing, 2009.

[2]. Transportation Bureau of MOR. "General Technical Scheme of CTCS-3 Train Control System”, China Railway Press, Beijing, 2008.

[3]. Transportation Bureau of MOR, “Test Cases for CTCS-3 Train Control System (v3.0),” Beijing: China Railway Publishing House, 2009.

[4]. Yu Liu, Tao Tang, "Research on the Method of Interoperability Test for the Onboard Equipment of CTCS-3 Train Control System of Chinese Railway”, Springer Berlin Heidelberg, 2012, 147:345-354. 
[5]. MULTITEL. Testing and Certification [EB/OL]. http : // www. multitel. Be / ertms / testing and - certification . php.

[6]. Zhang Yong, Qin Yan, Li Kai, Chen Jianqiu, The Method for Recognizing Driver Machine Interface Information of the Onboard Equipment in CTCS-3, China Railway Science, Vol.3, No.4, 92-100, 2010

[7]. Zhang Yong, Zhou Wenting, "Research on the automatic analysis and validation methodology of the CTCS-3 onboard equipment testing results”, 2014 Pacific-Asia Workshop on Computational Intelligence in Industrial Application (CIIA 2014). December 2014.pp437-439.

[8]. Zhang Yong, Zhang Bin. Design and implementation of CTCS-3 on-board equipment testing software based on multithreading [Z]. 2014 Pacific-Asia Workshop on Computational Intelligence in Industrial Application(CIIA 2014), December 2014.pp427-431. 